\title{
Digitizing the direct laryngoscopy experience: the economic way!!
}

\author{
Neha Hasija, Suniti Kale, and Kiran Kumar Girdhar \\ Department of Anaesthesia, Vardhman Mahavir Medical College and Safdarjung Hospital, New Delhi, India
}

Videolaryngoscopes have revolutionized the design of direct laryngoscopes and the process of endotracheal intubation [1]. Videolaryngoscopy is overcoming the limitations posed by direct laryngoscopy with increased angle of vision, visual feedback for the assistant as well as trainer, and the ability of still photography/video archiving facility. However, the cost of videolaryngoscopes becomes a limiting factor for their widespread use in developing countries and peripheral locations [2]. Incorporating an Android universal serial bus (USB) camera/borescope/snakescope into the direct laryngoscope blade and visualizing on your Android smartphone as a self-assembled device can prove to be an economic version of the videolaryngoscope $[3,4]$.

We used the $8 \mathrm{~mm}$ outer diameter Android endoscope (BESSSCOPE, China), which incorporates a waterproof $1 / 6$ inch, $5.5 \mathrm{~mm}$ VGA CMOS camera along with six light emitting diode lights with a resolution of 720P, which supports Android 4.0 or above along with Windows 2000/7/8/10/Vista. The video can be recorded in avi format and still photos in jpeg format. The viewing angle provided is 60 degrees, which is at par with commonly used videolaryngoscopes. The endoscope was disinfected by washing with soap and water followed by wiping with surgical spirit. The device was assembled by fixing a USB endoscope to the flange of the Macintosh as well as McCoy laryngoscope blade. The camera was fixed about $40 \mathrm{~mm}$ from the tip, levelled with the tip of bulb of the laryngoscope with the help of

Corresponding author: Neha Hasija, M.D., D.N.B., DESA

Department of Anaesthesia, Vardhman Mahavir Medical College and Safdarjung Hospital, A-77, First Floor, Malviya Nagar, New Delhi 110017, India

Tel: 91-8800450586, Fax: 91-8800450586

Email: hasijaneha@gmail.com

ORCID: https://orcid.org/0000-0001-6183-5026

Received: April 9, 2018.

Revised: May 7, 2018.

Accepted: May 14, 2018.

Korean J Anesthesiol 2018 December 71(6): 486-487

https://doi.org/10.4097/kja.d.18.00093 adhesive tape (Transpore $3 \mathrm{M}^{\mathrm{TM}}$ ) after checking the visual orientation (Fig. 1A). The view was recorded using phone/tablet with Android 4.0 and above, and using Scopecam application version 1.1 (Shenzhen wxl technology limited) downloaded from the Google Play Store.

We used this self-assembled device in both adult male and female patients using both Macintosh and McCoy blade. The view, clarity, and ease of intubation seem to be at par with the commercially available expensive C-MAC ${ }^{\circledR}$ video laryngoscope (Fig. 1B). This custom-made device can prove to be beneficial in low resource settings or remote locations providing the benefits of videolaryngoscopy to the direct laryngoscopy technique at a much lower cost. It can also help as a teaching tool for the trainers along with a great visual feedback to the assistant applying laryngeal manipulation. The need of integrating airway videos into electronic anesthesia chart as 'digital airway footprint' is also arising and use of this device can help in that regard [2].

Matters of concern while using this self-assembled device are the time taken to check visual orientation before fixing the endoscope camera (30-40 s), use of adhesive tapes repeatedly, and changing for every patient as well as space occupied by the camera head depending on size. These can probably be overcome by acquiring a custom-made blade that can hold the endoscope camera in the place of the bulb of the laryngoscope. Further work can be done in producing commercially available direct laryngoscope blades where the bulb can be removed and the endoscope camera can be fixed with ease without the use of adhesive tapes. The time is not far off when all anesthesia providers would be carrying this Android endoscope in their pockets along with their smartphones.

(c) This is an open-access article distributed under the terms of the Creative Commons Attribution Non-Commercial License (http://creativecommons.org/ licenses/by-nc/4.0/), which permits unrestricted non-commercial use, distribution, and reproduction in any medium, provided the original work is properly cited. 

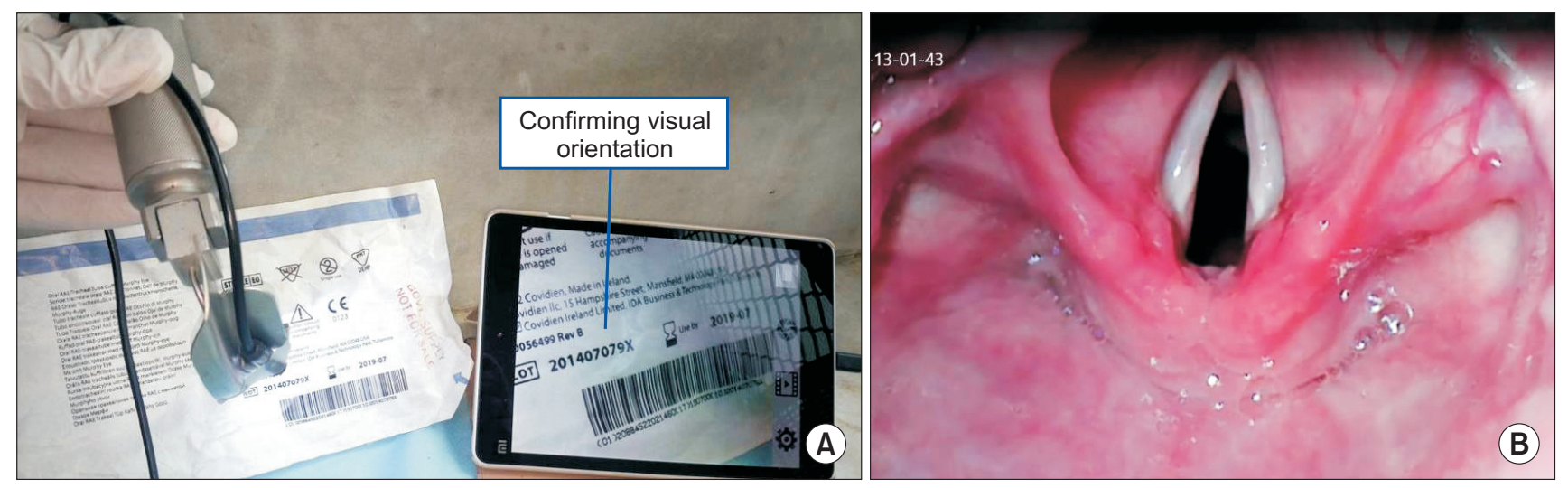

Fig. 1. (A) Figure demonstrating confirmation of the visual orientation of the USB endoscope and fixation with adhesive tape to the Macintosh laryngoscope. (B) Figure demonstrating laryngeal view utilizing the USB endoscope with the Macintosh laryngoscope.

\section{References}

1. Hurford WE. The video revolution: a new view of laryngoscopy. Respir Care 2010; 55: 1036-45.

2. Zaouter C, Calderon J, Hemmerling TM. Videolaryngoscopy as a new standard of care. Br J Anaesth 2015; 114: 181-3.

3. Karippacheril JG, Umesh G, Ramkumar V. Inexpensive video-laryngoscopy guided intubation using a personal computer: initial experience of a novel technique. J Clin Monit Comput 2014; 28: 261-4.

4. Karippacheril JG, Le Cong M. Videolaryngoscopy using an Android smartphone: a direct digital technique. Indian J Anaesth 2016; 60: 1435. 